\title{
VCPS: Vehicular Cyber-Physical Systems for Smart Road Services
}

\author{
Jaehoon (Paul) Jeong* and Eunseok Lee ${ }^{\dagger}$ \\ * Department of Software, Sungkyunkwan University, Republic of Korea \\ $\dagger$ Department of Computer Science \& Engineering, Sungkyunkwan University, Republic of Korea \\ Email: \{pauljeong,leees\}@skku.edu
}

\begin{abstract}
This paper proposes the design of Vehicular CyberPhysical Systems (called VCPS) for smart road services in vehicular networks. Our VCPS realizes mobile cloud computing services where vehicles themselves or mobile devices (e.g., smartphones and tablets of drivers or passengers in vehicles) play a role of both cloud server and cloud client. First, this paper describes the architecture of vehicular networks for VCPS and the delay modeling for the interactive navigation and accident prediction, such as a mobile node's travel delay along its navigation path in vehicular networks. Second, the paper explains two VCPS applications as smart road services through the vehicular cloud, such as (i) interactive navigation service for the efficient driving and (ii) pedestrian protection service against road accidents.
\end{abstract}

\section{INTRODUCTION}

Last decade, cloud computing has been researched and developed intensively, and then opened the new horizon of the Internet services [1]. This cloud computing has become a norm as mobile devices (such as smartphones and tablets) are popularly used as main computing devices for business, education, and entertainment. Since these mobile devices have limited computing power and storage, they can obtain almost unlimited computing power and storage from the cloud via wireless communications, such as 3G/4G-LTE [2] and WiFi.

Recently, mobile cloud computing has been introduced [3], regarding mobile devices (i.e., smartphones and tablets) as both cloud clients and cloud servers. These mobile devices perform sensing around the environments as mobile sensors and play a role of intermediate servers as computing nodes or storage nodes. This new paradigm for cloud computing will be expected to generate many useful services in many network areas, such as cellular networks, social networks, mobile ad hoc networks, vehicular networks, personal \& body networks, and mission critical networks. This paper focuses on the mobile cloud computing in vehicular networks for smart road services. The target applications are driving safety, navigation efficiency, and location-based services in road networks.

Vehicular Ad Hoc Networks (VANETs) have been researched and developed for the driving safety, driving efficiency, and entertainment services [4], [5]. This VANET has been realized by the technology of Dedicated Short Range Communications (DSRC) [6]. By DSRC, vehicles can communicate efficiently with other vehicles moving on either the same road segment or adjacent road segments at an intersection for the driving safety. This DSRC technology has been implemented by the standard of IEEE $802.11 \mathrm{p}$, which is an extension of IEEE 802.11a for vehicular networks. In addition, GPS navigation systems are popularly used by drivers for the efficient driving in the form of dedicated navigators [7] and smartphone navigator Apps [8]. Due to the DSRC standardization and navigator popularity, one natural research question is how to design Vehicular Cyber-Physical Systems by utilizing vehicles (equipped with DSRC device and navigator) as mobile cloud systems for smart road services.

In this paper, we propose the design of Vehicular CyberPhysical Systems (VCPS) using vehicular cloud computing as a branch of mobile cloud computing [3]. Our VCPS is managed by Traffic Control Center (TCC) [9] that maintains the road traffic conditions, vehicular traffic statistics (e.g., vehicle density and average speed) per road segment or intersection, and the navigation paths (called vehicle trajectories) of vehicles moving in road networks. Road-Side Units (RSUs) [10] are deployed as wireless gateway nodes that interconnect vehicular ad hoc networks and a wired network (i.e., the Internet), providing vehicles with the Internet connectivity to the TCC for the vehicular cloud services. Also, Relay Nodes (RNs) [11] are deployed as stand-alone, wireless packet holders that do not have the Internet connectivity for deployment cost saving, but can assist vehicles at intersections for either the data forwarding to/from the vehicular cloud or the information sharing for driving safety. In this paper, based on the VCPS, we design useful smart road services for the driving efficiency (e.g., interactive navigation) and the driving safety (e.g., pedestrian protection). As mobile cloud nodes, vehicles and smartphones can interact with each other via the vehicular cloud for these smart road services. Note that this paper is the compact version of our early magazine article [12].

The remaining of the paper is constructed as follows. Section II summarizes the related work. Section III describes the problem formulation for VCPS. Section IV explains the delay modeling for vehicle and pedestrian. Section $V$ describes two VCPS applications, such as interactive navigation and pedestrian protection. Finally, Section VI concludes the paper along with future work.

\section{RELATED WORK}

Nowadays, cloud computing has been realized and popularly used for a variety of Internet services. Cloud computing makes companies process their batch-oriented tasks through servers interconnected via networks in the scalable and elastic way [1]. As one of leading solution companies for cloud computing systems, VMware allows companies to run their own private cloud systems through the product of vCloud Suite [13]. Amazon runs Amazon Web Services (AWS) cloud 
service [14]. AWS provides cloud infrastructure for small businesses or persons according to the load of tasks with the corresponding charge for temporary lease of computing and storage resources. Mobile devices (e.g., smartphones and tablets) are used as main terminals for information retrieval and business transactions through the cloud. Google and Apple support various mobile services for mobile devices via their own cloud [15], [16].

The advent of mobile cloud computing is due to the popularity of mobile devices. Now mobile devices can run not only cloud client Apps, but also cloud servers or proxies for other mobile devices as mobile cloud [3]. The boundary of cloud clients and servers has broken down. As service models of mobile cloud computing, mobile devices play a role of (i) Mobile as a service consumer (MaaSC), (ii) Mobile as a service provider (MaaSP), and (iii) Mobile as a service broker (MaaSB). By being aware of user patterns and environment contexts, mobile devices can change their role dynamically to maximize the satisfaction of mobile users. Mobile devices can offload the task load of the cloud systems for other mobile devices as an intermediate cloud. This new paradigm will open new fascinating services in various networks, such as home networks, personal \& body networks, social networks, cellular networks, mobile ad hoc networks, vehicular networks, and mission critical networks. In this paper, we focus on the mobile cloud computing in vehicular networks.

Recently, vehicular networks have been intensively researched in various aspects, such as data forwarding schemes and Media Access Control (MAC) protocols. Data forwarding schemes are categorized into Vehicle-to-Infrastructure (V2I) data forwarding and Infrastructure-to-Vehicle (I2V) data forwarding. For the V2I data delivery, vehicular traffic statistics (e.g., vehicle inter-arrival time and average vehicle speed) are used in VADD [5]. In addition to the vehicular traffic statistics, vehicle trajectory (i.e., navigation path) is effectively in TBD [17] to improve forwarding performance in a privacypreserving manner. For the $\mathrm{I} 2 \mathrm{~V}$ data delivery, the vehicle trajectory of a destination vehicle is used in TSF [11] along with the vehicular traffic density. This multihop I2V data delivery has more challenge than the multihop V2I data delivery because the destination vehicle keeps moving over time, so a target point should be selected appropriately as a rendezvous point for the packet and destination vehicle. This target point selection is performed through the estimation of the destination vehicle's travel delay and the packet delivery delay. For the efficient data sharing among a multicast group of vehicles, TMA [18] is developed to extend the idea of TSF [11] considering the multiple target points for the multicast group vehicles. For the MAC protocols in vehicular networks, LMA [19] is a vehicle-to-vehicle (V2V) MAC protocol for the driving safety, using directional antenna and vehicle trajectory for the efficient wireless channel coverage. WPCF [20] is an efficient V2I and I2V MAC protocol using Point-Coordination Function (PCF) for wireless channel access, which proposes WAVE PCF where WAVE stands for Wireless Access in Vehicular Environments.

In this paper, with the emergence of mobile cloud computing and vehicular networks, we will design the architecture of vehicular networks for VCPS and the smart road services through VCPS. For the vehicular networks for VCPS, we will propose an organization of network systems consisting of TCC, RSUs, and RNs with appropriate V2I, I2V, and V2V data forwarding schemes. For the smart road services, we will suggest a feasible design of an interactive navigation service and a pedestrian protection service through the interaction between mobile devices and the vehicular cloud.

\section{PROBLEM Formulation}

In this section, for Vehicular Cyber-Physical Systems (VCPS), we describe our vehicular network architecture and then list up assumptions for our VCPS.

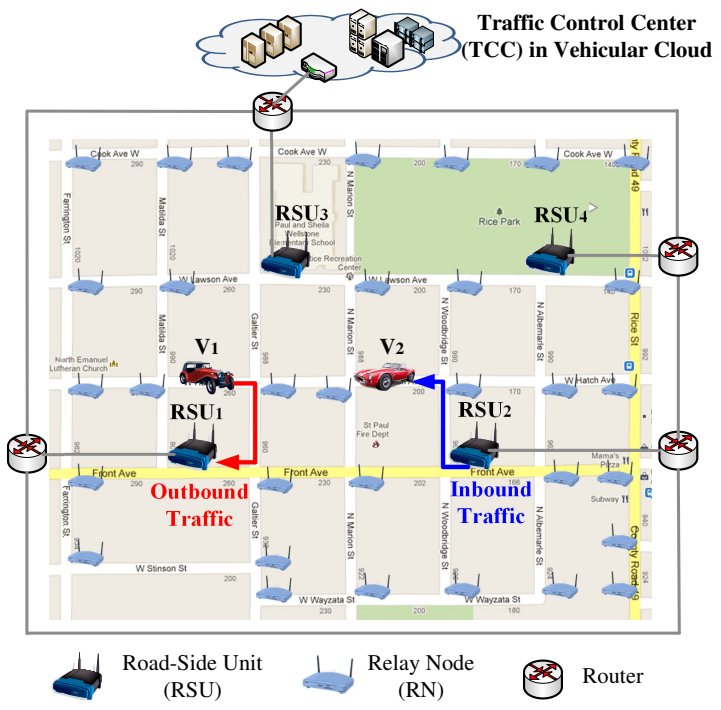

Fig. 1. Vehicular Networks for Vehicular Cyber-Physical Systems

\section{A. Vehicular Network Architecture}

Vehicular networks consist of the following system components:

- $\quad$ Traffic Control Center (TCC) [9] is a management node for vehicular cloud systems. As a trusted entity, TCC maintains the trajectories of vehicles for the location management for the data delivery toward the vehicles. These vehicle trajectories are not exposed to other vehicles for privacy concerns. In I2V data delivery, TCC determines which RSU will be the packet source node to deliver the packets to the moving destination vehicle(s) as shown in Fig. 1. It is assumed that TCC and RSUs are interconnected with each other through a wired network, such as the Internet.

- Road-Side Unit (RSU) [10] is a wireless node interconnecting vehicular ad hoc networks and a wired network. RSU has the DSRC communication, storage, and processing capability to forward packets from TCC to packet destination vehicles, as shown in Fig. 1. For the cost effectiveness, RSUs are sparsely deployed into the road network and are interconnected with each other through the wired network or wirelessly (as Mesh Network) [21]. Each RSU installation with power and wired network connectivity can cost as high as US\$5,000 [22]. 
- Relay Node (RN) [11] is a wireless stand-alone node as a temporary packet holder for the store-andforward of packets toward an intended direction in the road network. RN has the capability of DSRC communication, storage, and processing capability, but does not have the wired network connectivity for the cost saving, as shown in Fig. 1. This means that RNs do not have the direct, wired connectivity to either RSUs or TCC to save deployment cost. Also, it is assumed that RNs are not wirelessly connected to each other. However, in the case where RNs are wirelessly connected, we can regard the road segments among them as wirelessly covered by a Mesh Network consisting of those RNs. With a small number of RSUs, RNs are used to perform the reliable data delivery from RSU to the other RNs corresponding to the target points (i.e., packet destinations) by using intermediate vehicles as packet carriers, moving on road networks, as proved in our early work TSF [11]. One RN is assumed to be deployed at each intersection for the reliable forwarding, but we can handle the case where some intersections do not have their own RNs [11] by TSF.

- Vehicles have mobile devices (such as smartphones and tablets) or their dedicated on-board computers. As mobile sensors, vehicles can measure travel delay for each road segment along their travel path. For VANET, vehicles have DSRC device [6] along with other wireless communication devices, such as WiFi, WiMAX, 3G, and 4G-LTE [2]. Fig. 2 shows a smart vehicle with various devices for smart road services, such as mobile devices (e.g., smartphone and tablet), internal and external cameras, wireless communication devices (e.g., DSRC, 4G-LTE, 3G, $\mathrm{WiFi}$, and WiMAX), accelerometer, gyroscope, and vehicle computer. It is announced that major vehicle vendors (such as GM and Toyota) are planning to release vehicles with DSRC devices [23]. These DSRC vehicles play a role of packet forwarders and packet carriers until they can forward packets to a relay node or packet destination vehicle.

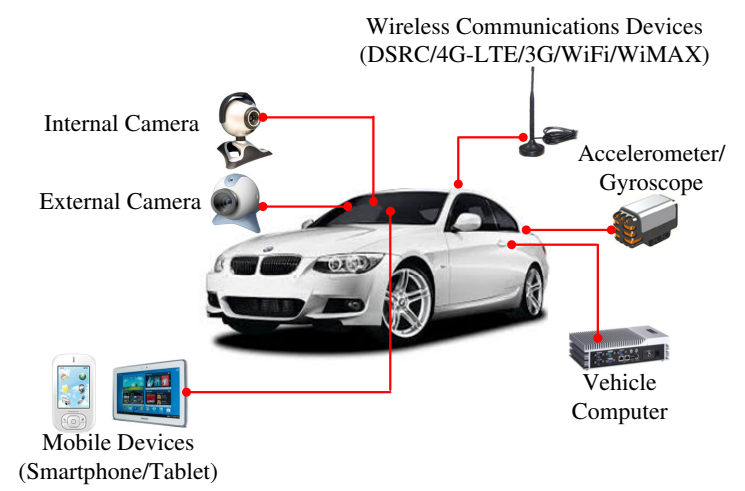

Fig. 2. Smart Vehicle

\section{B. Assumptions}

We have the following assumptions:

- Mobile devices (e.g., smartphones and tablets), TCC, RSUs, and RNs are installed with GPS-based nav- igation systems including digital road maps for location-based services (such as data forwarding or retrieval) [24]. Road traffic statistics, such as vehicle arrival rate $\lambda$ and average vehicle speed $v$ per road segment, are measured through mobile devices or loop-detectors. These traffic statistics can be used to produce metrics (e.g., link delay [11], [17]) for the data forwarding in road segments for smart road services.

- For smart road services, drivers or pedestrians voluntarily input their travel destination into their GPSbased navigation systems before their travel. This makes it possible for the vehicles to compute their future trajectory based on their current location and their final destination. Mobile devices (e.g., smartphones and tablets) in vehicles or in pockets regularly report their trajectory information and their current location to TCC through RSUs. This reporting can be performed, using the existing unicast forwarding schemes, such as TSF [11] and SADV [25].

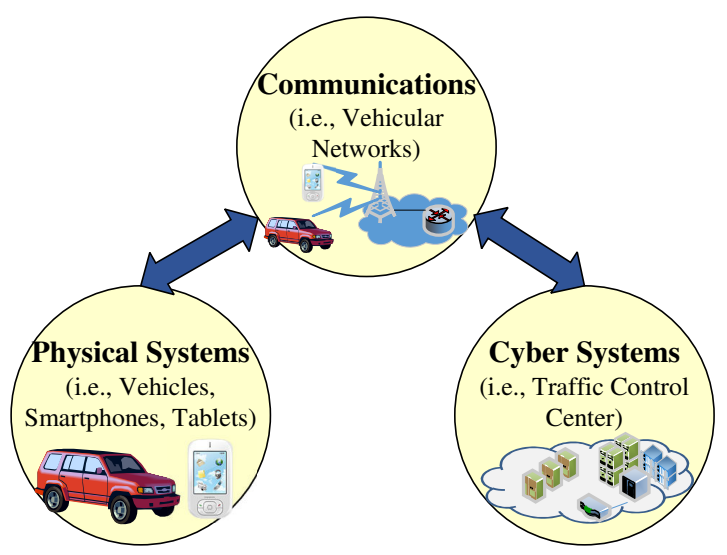

Fig. 3. Vehicular Cyber-Physical Systems

\section{The Concept of Vehicular Cyber-Physical Systems}

In this subsection, we formally define Cyber-Physical Systems (CPS) and Vehicular Cyber-Physical Systems (VCPS) and specify the target applications in VCPS.

Definition III.1 (CPS: Cyber-Physical Systems). Let CPS be the systems that are integrated by Physical Systems (following physical laws in continuous time domain) and Cyber Systems (following discrete mathematics in discrete time domain) via Communications.

Definition III.2 (VCPS: Vehicular Cyber-Physical Systems). Let VCPS be the systems that are integrated by Physical Systems in Road Networks and Cyber Systems in Vehicular Cloud via Wireless and Wired Communications, as a subset of $C P S$.

Fig. 3 illustrates the concept of VCPS using the interaction among system components, such as Physical Systems, Cyber Systems, and Communications. As shown in Fig. 3, VCPS consists of Physical Systems (e.g., Vehicles, Smartphones, and Tablets), Cyber Systems (e.g., Traffic Control Center), and Communications (e.g., Vehicular Networks). 
The VCPS takes advantages of the characteristics of road networks to design vehicular networks and services [11], [17], [18], such as (i) Predictable vehicle mobility, (ii) Road network layout, (iii) Vehicular traffic statistics, and (iv) Vehicle Trajectory. First, for Predictable vehicle mobility, vehicle moves along roadways with bounded speed. Second, for Road network layout, road network layout can be represented as a road map that can be reduced to a road network graph. Third, for Vehicular traffic statistics, vehicle inter-arrival time and average vehicle speed can be measured per road segment and average waiting time for traffic signal can be measured per intersection. Last, for Vehicle trajectory, vehicles follow the routes provided by GPS-based navigation systems for efficient driving. These characteristics are very important assets to design the vehicular networks (e.g., data forwarding schemes and media-access control protocols) and the vehicular services (e.g., interactive navigation and pedestrian protection). In next sections, considering these characteristics, we will show the delay modeling for mobile nodes (i.e., vehicle and pedestrian) and also VCPS applications (i.e., interactive navigation and pedestrian protection services).

\section{Travel Delay Modeling}

In this section, we model the travel delay of vehicle and pedestrian on a road segment and an End-to-End (E2E) travel path, such as vehicle trajectory and pedestrian trajectory. Note that this delay modeling originates from our early work TSF [11].

\section{A. Travel Delay on Road Segment}

Let $G=(V, E)$ be a road network graph where $V$ is a set of intersections and $E$ is a set of directed road segments. It is proved that the travel delay of one vehicle over a fixed distance in light-traffic vehicular networks follows the Gamma distribution [11] [26]. Thus, the travel delay through a road segment $i$ in the road network is defined as link travel delay $d_{i}$ such that $d_{i} \sim \Gamma\left(\kappa_{i}, \theta_{i}\right)$ where $\kappa_{i}$ is a shape parameter and $\theta_{i}$ is a scale parameter [27].

To calculate the parameters $\kappa_{i}$ and $\theta_{i}$, the mean $\mu_{i}$ and the variance $\sigma_{i}^{2}$ can be used for the link travel delay $d_{i}$ on the given road segment $e_{i} \in E$ [27]. The traffic statistics of $\mu_{i}$ and $\sigma_{i}^{2}$ are available from commercial navigation service providers (e.g., Garmin [24]). Let the mean of $d_{i}$ be $E\left[d_{i}\right]=\mu_{i}$ and the variance of $d_{i}$ be $\operatorname{Var}\left[d_{i}\right]=\sigma_{i}^{2}$. Thus, the formulas for $\kappa_{i}$ and $\theta_{i}$ are as follows [27]:

$$
\begin{gathered}
\theta_{i}=\frac{\operatorname{Var}\left[d_{i}\right]}{E\left[d_{i}\right]}=\frac{\sigma_{i}^{2}}{\mu_{i}} \\
\kappa_{i}=\frac{E\left[d_{i}\right]}{\theta_{i}}=\frac{\mu_{i}^{2}}{\sigma_{i}^{2}}
\end{gathered}
$$

In addition to the above mathematical model for link delay distribution on a road segment, our delay modeling can accommodate empirical measurements for the distribution of link delay. This empirical measurements can be performed by the periodical reports of mobile devices of vehicles or pedestrians (passing through a road segment or walking in a street) to the RSU taking charge of the road segment. Thus, a more accurate link travel delay distribution allows for a more accurate E2E travel delay distribution in the following subsection.

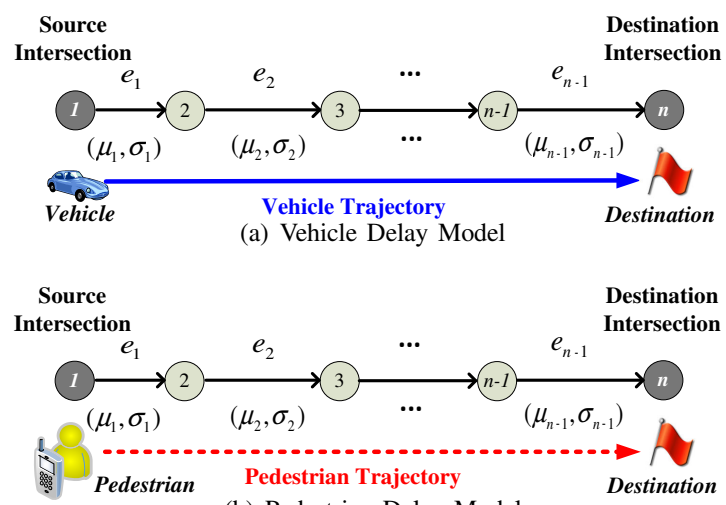

(b) Pedestrian Delay Model

Fig. 4. Travel Delay Model

\section{B. Travel Delay on End-to-End Path}

The End-to-End (E2E) travel delay in a road network can be modeled with the link delay model in Section IV-A [11]. As the link travel delay is modeled as the Gamma distribution of $d_{i} \sim \Gamma\left(\kappa_{i}, \theta_{i}\right)$ for road segment $i$, the E2E travel delay can be modeled with a sum of Gamma distributions of the link delays. Assume that as shown in Figs. 4(a) and 4(b), for two contiguous edges (e.g., $e_{1}$ and $e_{2}$ ) in a given E2E travel path, the intersection waiting delay from the first edge (e.g., $\left.e_{1}\right)$ to the second edge (e.g., $\left.e_{2}\right)$ is included in the first link travel delay (e.g., $\left.d_{1}\right)$.

Given an E2E travel path, it is assumed that the link travel delays of different road segments for the path are independent. With this assumption, the mean (or variance) of the E2E travel delay is approximately calculated as the sum of the means (or variances) of the link travel delays for the links along the E2E path. Assuming that the travel path consists of $n-1$ road segments, the mean and variance of the E2E travel delay $D$ are computed as follows:

$$
\begin{gathered}
E[D]=\sum_{i=1}^{n-1} E\left[d_{i}\right]=\sum_{i=1}^{n-1} \mu_{i} \\
\operatorname{Var}[D]=\sum_{i=1}^{n-1} \operatorname{Var}\left[d_{i}\right]=\sum_{i=1}^{n-1} \sigma_{i}^{2}
\end{gathered}
$$

With (3) and (4), the E2E travel delay $D$ is approximately modeled as a Gamma distribution as follows: $D \sim \Gamma\left(\kappa_{D}, \theta_{D}\right)$ where $\kappa_{D}$ and $\theta_{D}$ are calculated using $E[D]$ and $\operatorname{Var}[D]$ using the formulas of (1) and (2). Note that if a more accurate distribution for the E2E path is available from the measurements or another mathematical model, our travel delay model can use this distribution for the E2E travel delay estimation.

So far, we have explained our delay model for a mobile node's travel delay. In next section, we will design two smart road services using the delay models discussed in this section.

\section{VCPS APPLICATIONS}

In this section, we explain two applications as smart road services based on VCPS, such as (i) Interactive Navigation Service and (ii) Pedestrian Protection Service. 


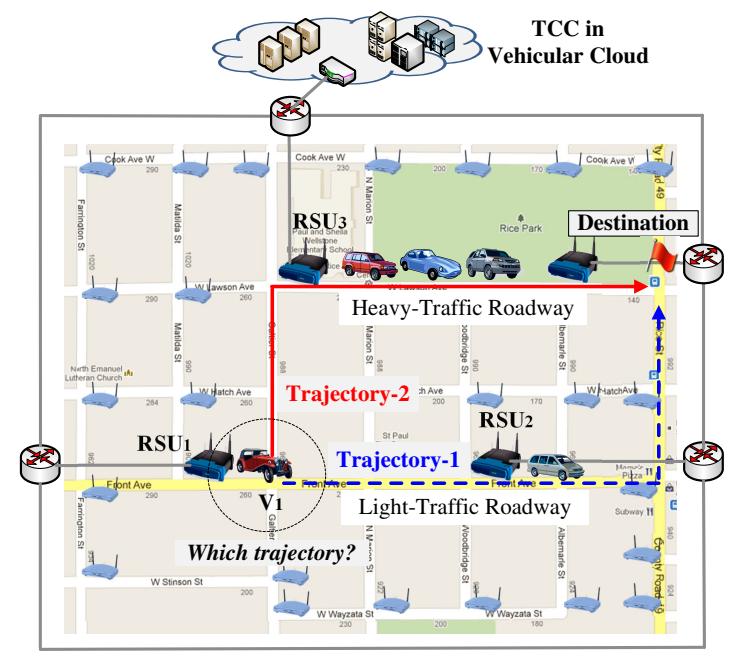

Fig. 5. Interactive Navigation through Vehicular Cloud

\section{A. Interactive Navigation Service}

With vehicular cloud, we can design an interactive navigation service for the more efficient driving for vehicles in road networks. The state-of-the-art navigation services [7], [8] are based on real-time road traffic, but they do not coordinate the traffic load balancing for the efficient road traffic flowing. For a better navigation service considering the traffic load balancing, the trajectories of the vehicles can be maintained by TCC in the vehicular cloud (as shown in Fig. 5). Thus, TCC will be able to predict which road segments will be highly congested in the near future. By this prediction, TCC can guide vehicles to take good alternative paths as their vehicle trajectories for the navigation.

Fig. 5 shows interactive navigation through the communication between vehicle $V_{1}$ and road-side unit $R S U_{1}$. Since $R S U_{1}$ is connected to $T C C$ via the Internet, $T C C$ can guide $V_{1}$ via $R S U_{1}$ to choose the best trajectory for its destination, considering both the current road traffic conditions and the future congestion in road segments.

Now we clarify the interaction between the vehicle and the vehicular cloud. It is assumed that Navigation Client is running on the vehicle as a smartphone App and Navigation Server is running on the vehicular cloud as a cloud server. The procedure for the interactive navigation service is as follows:

1) As Navigation Client, a vehicle with navigator contacts Navigation Server in TCC for navigating from its source to its destination. The navigation route request is performed by V2I data delivery scheme, such as TBD [17].

2) Navigation Server maintains road traffic matrices for a target road network graph to estimate both link travel delay and traffic congestion level per road segment in the graph.

3) With these matrices, Navigation Server computes an optimal route for the Navigation Client to experience the minimal travel delay, considering the future traffic conditions (i.e., traffic congestion levels) on the road segments in the target road network.

4) Navigation Server gives the optimal route to Navigation Client for navigation. The data delivery from
Navigation Server to Navigation Client is performed by I2V data delivery scheme, such as TSF [11]. This I2V data delivery toward a moving destination vehicle is possible because TCC maintains the trajectory of the vehicle as Navigation Client for the location management.

5) When receiving the route from Navigation Server, Navigation Client starts its travel along the guided route.

6) If Navigation Client goes out of the guided route, it repeats Steps 1 through 5 to get a new route from Navigation Server.

The research issue is how to construct and maintain the road traffic matrices for the estimation of link travel delay and traffic congestion level per road segment.

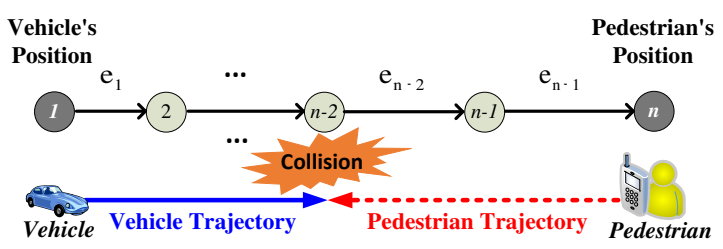

Fig. 6. Pedestrian Protection by Collision Prediction

\section{B. Pedestrian Protection Service}

Pedestrian protection is very important to reduce the fatality around school zones and downtown streets. Nowadays most of people are carrying a smartphone as either a pedestrian or a driver every day. This pedestrian protection can be performed through the communication between the smartphones of the pedestrian and the driver in the vehicle approaching the pedestrian. If two smartphones share their trajectories and motion vectors, it is feasible to tell the possibility that the pedestrian and the vehicle will collide by some mistake caused by either the pedestrian or the driver. When the vehicle is going to hit the pedestrian just in a couple of seconds, the smartphone of the driver will be able to notify the pedestrian of such a dangerous situation in the form of either voice or vibration.

Fig. 6 shows the pedestrian protection by the collision prediction of a vehicle and a pedestrian with the vehicle and pedestrian trajectories. This collision prediction can be performed by the delay modeling of the vehicle travel and the pedestrian travel, as discussed in Section IV.

Now we articulate the interaction between the pedestrian's smartphone and the vehicle's smartphone through the vehicular cloud. It is assumed that one Navigation Client is running on the pedestrian's smartphone and the vehicle's smartphone as a smartphone App, respectively and that Navigation Server is running on the vehicular cloud as a cloud server. Also, Navigation Agent is running on an RSU nearby the pedestrian for the sake of the effective information exchange among Navigation Clients along with Navigation Server as middle cloud [1], [3]. The procedure for the pedestrian protection service is as follows:

1) As Navigation Client, a smartphone with navigator periodically reports its location, direction, and speed to Navigation Server in TCC via adjacent RSUs during its travel from its source to its destination. The 
delivery of this location update message is performed by V2I data delivery scheme (such as TBD [17]) by using the smartphones of the neighboring vehicles or pedestrians as intermediate packet carriers.

2) Navigation Server maintains location and motion vector matrices for the smartphones of the pedestrians and vehicles in a target road network graph to predict the possible collision in the graph.

3) With these matrices, Navigation Server computes the collision probability for a pair of pedestrian and vehicle, considering the pedestrian trajectory and the vehicle trajectory along the road segments in the target road network.

4) For each pair with a high collision probability above the predefined threshold for accident avoidance, Navigation Server delivers the emergency message to both the vehicle and the pedestrian in one pair with a possible collision in the form of voice or vibration. This emergency message delivery must be performed within some short threshold (e.g., 0.1 second) by the cellular link through $4 \mathrm{G}-\mathrm{LTE} / 3 \mathrm{G}$ or I2V data delivery scheme (such as TSF [11]).

5) When receiving this notification from Navigation Server, Navigation Client immediately reacts to it by generating a special voice message or sound along with a special vibration to let the relevant pedestrian and the driver react to the dangerous situation promptly.

6) If Navigation Client goes out of the dangerous situation, it repeats Steps 1 through 5 for the pedestrian protection with Navigation Server.

In this pedestrian protection, it is important to minimize false negative and false positive for the collision between a vehicle and a pedestrian. Otherwise, the walking and driving will experience a lot of inconvenience by the misleading guidance for the pedestrian protection service.

\section{CONCLUSION}

In this paper, we proposed our design of Vehicular CyberPhysical Systems based on vehicular cloud. For the communications among mobile devices, vehicular networks need to support multiple wireless communications including DSRC. These vehicular networks consist of traffic control center, road-side units, relay nodes, and mobile devices (e.g., smartphones and tablets). To design smart road services, we first described our delay modeling for a mobile node's travel delay. We then explained two smart road services for the driving efficiency and safety, such as interactive navigation and pedestrian protection. As future work, we will investigate vehicular cloud considering the efficient battery consumption of mobile devices by using road-side units as middle cloud.

\section{ACKNOWLEDGMENT}

This research was supported by Next-Generation Information Computing Development Program through the National Research Foundation of Korea (NRF) funded by the Ministry of Science, ICT \& Future Planning (No. 2012033347) and by Faculty Research Fund, Sungkyunkwan University, 2013. This work was also partly supported by the IT R\&D program of MKE/KEIT [10041244, SmartTV 2.0 Software Platform] and by DGIST CPS Global Center.

\section{REFERENCES}

[1] M. A. et al., "A View of Cloud Computing," Communications of the ACM, vol. 53, no. 4, Apr. 2010.

[2] C. D. Monfreid, "The LTE Network Architecture - A Comprehensive Tutorial," Tech. Rep., 2009.

[3] D. Huang, T. Xing, and H. Wu, "Mobile Cloud Computing Service Models: A User-Centric Approach," IEEE Network, vol. 27, no. 5 , Sep. 2013.

[4] Q. Xu, R. Sengupta, and D. Jiang, "Design and Analysis of Highway Safety Communication Protocol in $5.9 \mathrm{GHz}$ Dedicated Short Range Communication Spectrum," in VTC. IEEE, Apr. 2003.

[5] J. Zhao and G. Cao, "VADD: Vehicle-Assisted Data Delivery in Vehicular Ad Hoc Networks," IEEE Transactions on Vehicular Technology, vol. 57, no. 3, pp. 1910-1922, May 2008.

[6] Y. L. Morgan, "Notes on DSRC \& WAVE Standards Suite: Its Architecture, Design, and Characteristics," IEEE Communications Surveys \& Tutorials, vol. 12, no. 4, pp. 504-518, Oct. 2010.

[7] Garmin, "Dedicated Navigator," http://www.garmin.com.

[8] Waze, "Smartphone App for Navigator," https://www.waze.com.

[9] Philadelphia Department of Transportation, "Traffic Control Center," http://philadelphia.pahighways.com/philadelphiatcc.html.

[10] A. Abdrabou and W. Zhuang, "Probabilistic Delay Control and Road Side Unit Placement for Vehicular Ad Hoc Networks with Disrupted Connectivity," IEEE Journal on Selected Areas in Communications, vol. 29, no. 1, pp. 129-139, Jan. 2011.

[11] J. Jeong, S. Guo, Y. Gu, T. He, and D. Du, "Trajectory-Based Statistical Forwarding for Multihop Infrastructure-to-Vehicle Data Delivery," IEEE Transactions on Mobile Computing, vol. 11, no. 10, pp. 15231537, Oct. 2012.

[12] J. Jeong and E. Lee, "Vehicular Cyber-Physical Systems for Smart Road Networks," KICS Information and Communications Magazine, vol. 31, no. 3, pp. 103-116, Mar. 2014.

[13] VMware, "vCloud Suite," http://www.vmware.com.

[14] Amazon, "Amazon Web Services," http://aws.amazon.com.

[15] Google, "Google Cloud Platform," https://cloud.google.com.

[16] Apple, "Apple iCloud," http://www.apple.com/icloud/.

[17] J. Jeong, S. Guo, Y. Gu, T. He, and D. Du, "Trajectory-Based Data Forwarding for Light-Traffic Vehicular Ad-Hoc Networks," IEEE Transactions on Parallel and Distributed Systems, vol. 22, no. 5, pp. 743-757, May 2011.

[18] J. Jeong, T. He, and D. Du, "TMA: Trajectory-based Multi-Anycast Forwarding for Efficient Multicast Data Delivery in Vehicular Networks," Elsevier Computer Networks, vol. 57, no. 13, pp. 2549-2563, Sep. 2013.

[19] K.-T. Feng, "LMA: Location- and Mobility-Aware Medium-Access Control Protocols for Vehicular Ad Hoc Networks Using Directional Antenna," IEEE Transactions on Vehicular Technology, vol. 56, no. 6, pp. 3324-3336, Nov. 2007.

[20] J.-M. Chung, M. Kim, Y.-S. Park, M. Choi, S. W. Lee, and H. S. Oh, "Time Coordinated V2I Communications and Handover for WAVE Networks," IEEE Journal on Selected Areas in Communications, vol. 29, no. 3, pp. 545-558, Mar. 2011.

[21] N. Banerjee, M. D. Corner, D. Towsley, and B. N. Levine, "Relays, Base Stations, and Meshes: Enhancing Mobile Networks with Infrastructure," in MOBICOM. ACM, Sep. 2008.

[22] Jupiter Research, "Municipal Wireless: Partner to Spread Risks and Costs While Maximizing Benefit Opportunities," Tech. Rep., Jun. 2005.

[23] General Motors (GM), "Vehicle-to-Vehicle (V2V) Communications," http://www.gm.com/experience/technology/research/overview/isl/vcim. jsp.

[24] Garmin Ltd., "Garmin Traffic," http://www8.garmin.com/traffic/.

[25] Y. Ding, C. Wang, and L. Xiao, "A Static-Node Assisted Adaptive Routing Protocol in Vehicular Networks," in VANET. ACM, Sep. 2007

[26] A. Polus, "A Study of Travel Time and Reliability on Arterial Routes," Transportation, vol. 8, no. 2, pp. 141-151, Jun. 1979.

[27] M. DeGroot and M. Schervish, Probability and Statistics (3rd Edition). Addison-Wesley, 2001. 